PROCEEDINGS OF THE

AMERICAN MATHEMATICAL SOCIETY

Volume 133, Number 5, Pages 1427-1435

S 0002-9939(04)07655-5

Article electronically published on November 1, 2004

\title{
SPECTRALLY BOUNDED $\phi$-DERIVATIONS ON BANACH ALGEBRAS
}

\author{
TSIU-KWEN LEE AND CHENG-KAI LIU
}

(Communicated by Joseph A. Ball)

\begin{abstract}
Applying the density theorem on algebras with $\phi$-derivations, we show that if a $\phi$-derivation $\delta$ of a unital Banach algebra $A$ is spectrally bounded, then $[\delta(A), A] \subseteq \operatorname{rad}(A)$. Also, $\delta(A) \subseteq \operatorname{rad}(A)$ if and only if $\sup \left\{r\left(z^{-1} \delta(z)\right) \mid z \in A\right.$ is invertible $\}<\infty$, where $\bar{r}(a)$ denotes the spectral radius of $a \in A$.
\end{abstract}

\section{INTRODUCTION}

Throughout, unless specially stated, $A$ always denotes a unital Banach algebra over the complex field $\mathbf{C}$. We denote by $\operatorname{rad}(A)$ the Jacobson radical of $A$ and by $r(x)$ the spectral radius of $x \in A$. Also, let $Q(A)$ be the set of all quasinilpotent elements of $A$. A derivation $d: A \rightarrow A$ is a linear mapping satisfying $d(x y)=$ $d(x) y+x d(y)$ for all $x, y \in A$; an inner derivation is a derivation of the form $d(x)=a x-x a$ for some $a \in A$, and a generalized inner derivation is one of the form $d(x)=a x-x b$ for some $a, b \in A$. All derivations or automorphisms of $A$ are always assumed to be linear. A linear mapping $T: A \rightarrow A$ is called spectrally bounded if there exists $M \geq 0$ such that $r(T(x)) \leq M r(x)$ for all $x \in A$. In addition, if $M=0$ (i.e., $T(A) \subseteq Q(A)$ ), $T$ is called spectrally infinitesimal. Clearly, we have the following implications:

$$
T(A) \subseteq \operatorname{rad}(A) \Rightarrow T \text { spectrally infinitesimal } \Rightarrow T \text { spectrally bounded. }
$$

However, none of the above reverse implications is true in general. The SingerWermer Theorem [14] states that $d(A) \subseteq \operatorname{rad}(A)$ if $d$ is a bounded derivation of a commutative Banach algebra $A$. In 1988, Thomas [15] proved the same conclusion without the bounded assumption on the derivation $d$. The noncommutative SingerWermer conjecture states that each derivation $d$ on a Banach algebra $A$ leaves each primitive ideal invariant, which is equivalent to $d(A) \subseteq \operatorname{rad}(A)$ if $[d(a), a] \in \operatorname{rad}(A)$ for all $a \in A$ [12, Theorem 1.8]. Also, $d$ spectrally infinitesimal always implies that $d(A) \subseteq \operatorname{rad}(A)$ for $d$ bounded [11] or arbitrary [16.

In [1] Brešar proved that every spectrally bounded inner derivation maps into the radical. Later, Curto and Mathieu [7] characterized spectrally bounded generalized inner derivations. Finally, Brešar and Mathieu [4] proved that a derivation $d$ of $\mathrm{A}$ is spectrally bounded if and only if $d(A) \subseteq \operatorname{rad}(A)$. In [3] Brešar proved an extension

Received by the editors September 10, 2003 and, in revised form, January 14, 2004.

2000 Mathematics Subject Classification. Primary 47B48, 46H15.

Key words and phrases. Radical, $\phi$-derivation, Banach algebra, spectrally bounded mapping.

(C)2004 American Mathematical Society Reverts to public domain 28 years from publication 
of the Jacobson density theorem for Banach algebras with automorphisms. As its applications, automorphisms $\phi$ of Banach algebras such that $\phi-1$ is spectrally bounded are characterized (see [3, Theorems 3.1 and 3.3]).

Let $\phi$ be an automorphism of $A$. By a $\phi$-derivation of $A$ we mean an additive map $\delta: A \rightarrow A$ such that $\delta(x y)=\phi(x) \delta(y)+\delta(x) y$ for all $x, y \in A$. Clearly, derivations and the maps $\phi-1$ are $\phi$-derivations. In the present paper we study $\phi$-derivations to unify both derivations and maps of type $\phi-1$ on Banach algebras. Applying the density theorem on algebras with $\phi$-derivations we will prove the following two main results:

Theorem 1.1. Let $\delta$ be a spectrally bounded $\phi$-derivation on a unital Banach algebra $A$. Then $[\delta(A), A] \subseteq \operatorname{rad}(A)$.

Theorem 1.2. Let $\delta$ be a $\phi$-derivation of a unital Banach algebra $A$. Then $\delta(A) \subseteq$ $\operatorname{rad}(A)$ if and only if $\sup \left\{r\left(z^{-1} \delta(z)\right) \mid z \in A\right.$ is invertible $\}<\infty$.

Remarks. (1) Theorem 1.1 was proved by Brešar for $\delta=\phi-1$ [3, Theorem 3.1]. Moreover, in this case, $[\delta(A), A] \subseteq \operatorname{rad}(A)$ implies that $\delta$ is spectrally bounded. However, the general case is unknown even when $\delta$ is a derivation. Indeed, in view of [9, Theorem 1.2] the condition that $[\delta(a), a] \in \operatorname{rad}(A)$ for all $a \in A$ implies that $[\delta(A), A] \subseteq \operatorname{rad}(A)$. Hence, if the reverse implication of Theorem 1.1 is true for the derivation case, then $\delta$ is spectrally bounded and so, by Brešar and Mathieu's theorem [4], $\delta(A) \subseteq \operatorname{rad}(A)$ follows. Thus the noncommutative Singer-Wermer conjecture is true.

(2) Theorem 1.2 has been obtained by Brešar and Mathieu [4, Theorem 2.6] for derivations and by Brešar [3, Theorem 3.3] for the $\phi$-derivation $\phi-1$.

\section{Preliminary Results}

In this section we will quote some results given in [10, which will be used in the sequel. For simplicity, we only state its special form required here. Throughout this section, unless specially stated, we make the following assumptions:

Let $A$ be an algebra over a field $k$ with ${ }_{A} E$ an irreducible left $A$-module, and let $D=\operatorname{End}\left({ }_{A} E\right)$ be the associated division algebra of ${ }_{A} E$. Let $\phi$ be a $k$-linear automorphism of $A$. By the Jacobson density theorem, $A$ acts densely on $E_{D}$. We define the map $\pi: A \rightarrow \operatorname{End}\left(E_{D}\right)$ by the left multiplications:

$$
a \in A \mapsto \pi(a) \in \operatorname{End}\left(E_{D}\right) \text {, where } \pi(a) m=a m \text { for all } m \in E .
$$

By a $\phi$-derivation $\delta: A \rightarrow \operatorname{End}\left(E_{D}\right)$ we mean that $\delta$ is a $k$-linear map satisfying $\delta(x y)=\pi \phi(x) \delta(y)+\delta(x) \pi(y)$ for all $x, y \in A$. We remark that if $\delta: A \rightarrow A$ is a $k$-linear $\phi$-derivation, then $\delta$ canonically induces a $\phi$-derivation $\bar{\delta}: A \rightarrow \operatorname{End}\left(E_{D}\right)$ by the rule: $a \in A \mapsto \bar{\delta}(a)=\pi \delta(a)$.

Definition. (1) A $k$-linear automorphism $\phi$ of $A$ is called $E$-inner if there exists an invertible element $S \in \operatorname{End}_{k}(E)$ such that $\pi \phi(a)=S^{-1} \pi(a) S$ for all $a \in A$. Otherwise, it is called $E$-outer.

(2) A $\phi$-derivation $\delta: A \rightarrow \operatorname{End}\left(E_{D}\right)$ is called $E$-inner if there exists $T \in$ $\operatorname{End}_{k}(E)$ such that $\delta(a)=\pi \phi(a) T-T \pi(a)$ for all $a \in A$. Otherwise, it is called $E$-outer. Moreover, if a $\phi$-derivation $\delta: A \rightarrow A$ is called $E$-inner ( $E$-outer) if its induced $\phi$-derivation $\bar{\delta}: A \rightarrow \operatorname{End}\left(E_{D}\right)$ is $E$-inner (resp. $E$-outer).

The following two theorems are special forms of [10, Theorems 2.2 and 2.3] (by considering the case of left modules). 
Theorem 2.1. A $\phi$-derivation $\delta: A \rightarrow \operatorname{End}\left(E_{D}\right)$, where $\phi$ is $E$-inner, is E-outer if and only if given finitely many $D$-independent $x_{i} \in E$ and arbitrary $y_{i}, z_{i} \in E$, where $1 \leq i \leq n$, there exists $a \in A$ such that $\pi(a) x_{i}=y_{i}, \delta(a) x_{i}=z_{i}$ for $1 \leq i \leq n$.

Theorem 2.2. $A$-derivation $\delta: A \rightarrow \operatorname{End}\left(E_{D}\right)$, where $\phi$ is $E$-outer, is E-outer if and only if given finitely many $D$-independent $x_{i} \in E$ and arbitrary $y_{i}, z_{i}, w_{i} \in E$, where $1 \leq i \leq n$, there exists $a \in A$ such that $\pi(a) x_{i}=y_{i}, \pi \phi(a) x_{i}=z_{i}$, and $\delta(a) x_{i}=w_{i}$ for $1 \leq i \leq n$.

\section{Proofs}

We are now ready to proceed with our proofs of the theorems stated in the first section. We begin with the following:

Lemma 3.1. Let $\delta$ be a spectrally bounded $\phi$-derivation on $A$. Then $\operatorname{rad}(A)$ is invariant under $\delta$.

Proof. Let $x \in \operatorname{rad}(A)$ and $a \in A$. Then $\phi^{-1}(a) x \in \operatorname{rad}(A)$, and so $r\left(\phi^{-1}(a) x\right)=0$. Since $\delta$ is spectrally bounded, applying the fact that $\delta\left(\phi^{-1}(a)\right) x \in \operatorname{rad}(A)$ we have

$$
0=r\left(\delta\left(\phi^{-1}(a) x\right)\right)=r\left(a \delta(x)+\delta\left(\phi^{-1}(a)\right) x\right)=r(a \delta(x)),
$$

implying that $a \delta(x)$ is quasi-nilpotent and hence is also quasi-invertible in $A$. Thus $\delta(x) \in \operatorname{rad}(A)$. This proves that $\delta(\operatorname{rad}(A)) \subseteq \operatorname{rad}(A)$, as asserted.

Let $A$ be a Banach algebra with $\phi$ a linear automorphism, and let $\delta: A \rightarrow A$ be a $\phi$-derivation. Suppose that $(\pi, E)$ is a continuous irreducible representation of $A$. It is well known that $\operatorname{End}\left({ }_{A} E\right)=\mathbf{C}$. Applying the notion given in $\S 2$ we have: (1) An automorphism $\phi$ of $A$ is $E$-inner if there exists an invertible $S \in \operatorname{End}_{\mathbf{C}}(E)$ such that $\pi \phi(a)=S^{-1} \pi(a) S$ for all $a \in A$. (2) A $\phi$-derivation $\delta$ on $A$ is called $E$-inner if there exists $T \in \operatorname{End}_{\mathbf{C}}(E)$ such that $\pi \delta(a)=\pi \phi(a) T-T \pi(a)$ for all $a \in A$. We need the following result in the proof of Theorem 1.1.

Lemma 3.2. Let $\delta$ be a $\phi$-derivation of $A$, and let $(\pi, E)$ be a continuous irreducible representation of $A$ with $\operatorname{dim}_{\mathbf{C}} E \geq 2$. Suppose that $\delta$ is $E$-outer. Then there exist $\mathbf{C}$-independent vectors $u, v \in E$ and $x, y \in A$ such that

$$
\begin{aligned}
& \pi \delta(x) v=0, \pi \phi(x) u=v, \pi(x) v=0 \text { and } \\
& \pi \delta(y) v=u, \pi \phi(y) v=0, \pi \phi(y) u=0, \pi(y) v=0 .
\end{aligned}
$$

Proof. Suppose first that $\phi$ is $E$-outer. Choose $\mathbf{C}$-independent $u, v \in E$. In view of Theorem 2.2, we can choose $x, y \in A$ satisfying (3.1). Suppose next that $\phi$ is $E$-inner. Thus, by definition, there exists an invertible element $S \in \operatorname{End}_{\mathbf{C}}(E)$ such that $\pi \phi(a)=S^{-1} \pi(a) S$ for all $a \in A$. We can choose $\mathbf{C}$-independent $u, v \in E$ such that $S u$ and $v$ are $\mathbf{C}$-independent. Indeed, we first choose a nonzero $u \in E$. If $S u=\beta u$ for some $\beta \in \mathbf{C}$, we are done by choosing an arbitrary $v \notin \mathbf{C} u$. Otherwise, $S u \notin \mathbf{C} u$, and let $v=u+S u$ in this case. Applying Theorem 2.1 we can choose $x \in A$ such that

$$
\pi \delta(x) v=0, \pi(x) S u=S v, \text { and } \pi(x) v=0 .
$$

Thus $\pi \phi(x) u=S^{-1} \pi(x) S u=v$. Since $u$ and $v$ are $\mathbf{C}$-independent, by Theorem 2.1 there exists $y \in A$ such that $\pi \delta(y) v=u$ and $\pi(y)$ maps the $\mathbf{C}$-subspace $\mathbf{C} v+\mathbf{C} S u+$ $\mathbf{C} S v$ into zero. But $S \pi \phi(y)=\pi(y) S$; this means that $\pi \phi(y) v=0, \pi \phi(y) u=0$, and $\pi(y) v=0$. This proves the lemma. 
Let $R_{i} \in \operatorname{Hom}_{F}(U, V), 1 \leq i \leq n$, where $U$ and $V$ are two vector spaces over a field $F$. We say that the $R_{i}$ 's are locally dependent if, for each $u \in U$, the set $\left\{R_{i} u \mid 1 \leq i \leq n\right\}$ is $F$-dependent. The following theorem is given in [5, Theorem 2.4]. We state its form needed here.

Theorem 3.3 (Brešar and Šemrl [5]). Let $R_{i} \in \operatorname{Hom}_{F}(U, V), i=1,2,3$, where $U$ and $V$ are two vector spaces over an infinite field $F$, char $F \neq 2$. Suppose that $R_{1}, R_{2}$, and $R_{3}$ are locally dependent but not $F$-dependent. Then either $\operatorname{dim}_{F} R_{i} U$ $\leq 3$ for all $i$ or there exists $P \in \operatorname{End}_{F}(V)$ an idempotent of rank 1 such that

$$
\operatorname{dim} \operatorname{span}\left\{\left(I_{V}-P\right) R_{1},\left(I_{V}-P\right) R_{2},\left(I_{V}-P\right) R_{3}\right\}=1 .
$$

We are now in a position to give the

Proof of Theorem 1.1. We may assume that $A$ is semisimple. Indeed, note that $\phi(\operatorname{rad}(A))=\operatorname{rad}(A)$. Thus $\phi$ canonically induces an automorphism $\bar{\phi}$ on $A / \operatorname{rad}(A)$. By Lemma 3.1, $\delta(\operatorname{rad}(A)) \subseteq \operatorname{rad}(A)$. We set $\bar{x}=x+\operatorname{rad}(A) \in A / \operatorname{rad}(A)$ for $x \in A$. Then $\delta$ also induces a $\bar{\phi}$-derivation $\bar{\delta}$ on $A / \operatorname{rad}(A)$ by the rule: $\bar{\delta}(\bar{x})=\overline{\delta(x)}$ for all $x \in A$. Moreover,

$$
r(\bar{\delta}(\bar{x}))=r(\delta(x)) \leq M r(x)=M r(\bar{x})
$$

for all $x \in A$. That is, $\bar{\delta}$ is still a spectrally bounded $\bar{\phi}$-derivation on $A / \operatorname{rad}(A)$, a semisimple Banach algebra. Thus $[\bar{\delta}(A / \operatorname{rad}(A)), A / \operatorname{rad}(A)]=0$, and so $[\delta(A), A] \subseteq$ $\operatorname{rad}(A)$, as asserted. So we can assume from the start that $A$ is semisimple.

By [8] and [6. Corollary 4.3], both $\phi$ and $\delta$ are continuous. Let $(\pi, E)$ be a continuous irreducible representation of $A$. Clearly, it suffices to prove that $[\delta(A), A] \subseteq \operatorname{ker}(\pi)$ for each $(\pi, E)$. If $\operatorname{dim}_{\mathbf{C}} E=1$, then $[\pi(A), \pi(A)]=0$ and so $[A, A] \subseteq \operatorname{ker}(\pi)$, as asserted. We now consider the case that $\operatorname{dim}_{\mathbf{C}} E \geq 2$. The aim is to prove that $\pi \delta(A) E=0$.

Case 1. Suppose that $\delta$ is $E$-outer. Let $u, v \in E$ and $x, y \in A$ be given as in Lemma 3.2. That is,

$$
\pi \delta(y) v=u, \pi \phi(y) v=0, \pi \phi(y) u=0, \text { and } \pi(y) v=0 .
$$

We set $z_{n}=e^{n y}$ for $n \geq 0$. Applying the continuity of both $\pi$ and $\delta$ we have

$$
\begin{gathered}
\pi\left(z_{n}\right) v=v, \pi \phi\left(z_{n}\right) v=v, \text { and } \\
\pi \delta\left(z_{n}\right) v=\left(\delta(1)+\pi(n \delta(y))+\frac{1}{2 !}\left(\pi \delta\left(n^{2} y^{2}\right)+\cdots\right) v=n u,\right.
\end{gathered}
$$

since

$$
\pi \delta\left(n^{k} y^{k}\right) v=n^{k}\left((\pi \phi(y))^{k-1} \pi \delta(y)+\pi \delta\left(y^{k-1}\right) \pi(y)\right) v=0
$$

for $k \geq 2$. On the other hand, by (3.1) we have $\pi \delta(x) v=0, \pi \phi(x) u=v$ and $\pi(x) v=0$. We compute

$$
\begin{aligned}
& \pi \delta\left(z_{n}^{-1} x z_{n}\right) v \\
= & \pi\left(\phi\left(z_{n}^{-1}\right) \phi(x) \delta\left(z_{n}\right)\right) v+\pi\left(\phi\left(z_{n}^{-1}\right) \delta(x) z_{n}\right) v+\pi\left(\delta\left(z_{n}^{-1}\right) x z_{n}\right) v=n v .
\end{aligned}
$$

Then $\operatorname{Mr}(x)=\operatorname{Mr}\left(z_{n}^{-1} x z_{n}\right) \geq r\left(\delta\left(z_{n}^{-1} x z_{n}\right)\right) \geq r\left(\pi \delta\left(z_{n}^{-1} x z_{n}\right)\right) \geq n$ for all $n \geq 1$, a contradiction.

Case 2. Assume that $\delta$ is $E$-inner, but $\phi$ is $E$-outer. In this case, there exists $T \in \operatorname{End}_{\mathbf{C}}(E)$ such that $\pi \delta(x)=\pi \phi(x) T-T \pi(x)$ for all $x \in A$. Suppose first that $T$ is not a scalar. Choose $v \in E$ such that $T v$ and $v$ are $\mathbf{C}$-independent. In view of 
[3, Theorems 2.1 and 1.2], there exist invertible $z_{n} \in A$ for each $n \geq 1$ and $x \in A$ such that

$$
\begin{gathered}
\pi \phi\left(z_{n}\right) T v=n v, \pi \phi\left(z_{n}\right) v=T v, \pi\left(z_{n}\right) v=v, \\
\pi \phi(x) v=T v, \text { and } \pi(x) v=0 .
\end{gathered}
$$

Then $\pi \phi\left(z_{n}^{-1}\right) T v=v$, and so

$$
\pi \delta\left(z_{n}^{-1} x z_{n}\right) v=\pi\left(\phi\left(z_{n}^{-1}\right) \phi(x) \phi\left(z_{n}\right)\right) T v-T \pi\left(z_{n}^{-1} x z_{n}\right) v=n v .
$$

This derives a contradiction as in Case 1. Hence, we assume that $T$ is a nonzero scalar. Without loss of generality, we may assume that $T=1$. Choose $\mathbf{C}^{-}$ independent $u, v \in E$. By [3, Theorems 2.1 and 1.2] again, there exist invertible $z_{n} \in A$ for each $n \geq 1$ and $x \in A$ such that

$$
\begin{gathered}
\pi\left(z_{n}\right) v=v, \pi\left(z_{n}\right) u=u, \pi \phi\left(z_{n}\right) v=u, \pi \phi\left(z_{n}\right) u=v-n u, \\
\pi(x) v=u, \pi \phi(x) v=0, \text { and } \pi \phi(x) u=v .
\end{gathered}
$$

Then $\pi \delta\left(z_{n}^{-1} x z_{n}\right) v=\pi\left(\phi\left(z_{n}^{-1}\right) \phi(x) \phi\left(z_{n}\right)-z_{n}^{-1} x z_{n}\right) v=n v$, a contradiction.

Case 3. Assume that both $\delta$ and $\phi$ are $E$-inner. Thus there exist $S, T \in \operatorname{End}_{\mathbf{C}}(E)$ with $S$ invertible such that

$$
\pi \delta(x)=\pi \phi(x) T-T \pi(x) \text { and } \pi \phi(x)=S^{-1} \pi(x) S \text { for all } x \in A .
$$

By the above we see that

$$
\pi \delta(x)=S^{-1}[\pi(x), U] \text { for all } x \in A,
$$

where $U=S T$. Note that if $U$ is a scalar, then $\pi \delta(A) E=0$ and so $\delta(A) \subseteq \operatorname{ker}(\pi)$. We are done in this case. Assume that $U$ is not a scalar.

Suppose there exists a vector $v \in E$ such that $S v, U v$ and $v$ are $\mathbf{C}$-independent. In view of [13, p. 36], for $n \geq 0$ we choose an invertible element $z_{n} \in A$ satisfying

$$
\pi\left(z_{n}\right) S v=S v, \pi\left(z_{n}\right) U v=v+n S v, \text { and } \pi\left(z_{n}\right) v=U v .
$$

Applying the Jacobson density theorem, we can choose $x \in A$ such that $\pi(x) v=0$, $\pi(x) U v=0$ and $\pi(x) S v=S v$. We now compute

$$
\begin{aligned}
\pi \delta\left(z_{n}^{-1} x z_{n}\right) v & =\left(S^{-1} \pi\left(z_{n}^{-1}\right) \pi(x) \pi\left(z_{n}\right) U-S^{-1} U \pi\left(z_{n}^{-1}\right) \pi(x) \pi\left(z_{n}\right)\right) v \\
& =S^{-1} \pi\left(z_{n}^{-1}\right) \pi(x) \pi\left(z_{n}\right) U v=n v .
\end{aligned}
$$

This derives a contradiction. Thus we have proved that, for each $v \in E$, the three vectors $S v, U v$ and $v$ are $\mathbf{C}$-dependent. That is, $I_{E}, S, U$ are locally dependent.

In view of Theorem 3.3, either $\operatorname{dim}_{\mathbf{C}} E \leq 3$, or there exists $P \in \operatorname{End}_{\mathbf{C}}(E)$ an idempotent of rank 1 such that $\operatorname{dim} \operatorname{span}\left\{I_{E}-P,\left(I_{E}-P\right) S,\left(I_{E}-P\right) U\right\}=1$, or $I_{E}, U$ and $S$ are $\mathbf{C}$-dependent. For brevity, we denote $I=I_{E}$.

Consider first that $\operatorname{dim}_{\mathbf{C}} E \leq 3$.

(I) Suppose that $\operatorname{dim}_{\mathbf{C}} E=3$. In this case, $\pi(A) \cong \mathrm{M}_{3}(\mathbf{C})$. For brevity, we write $\delta(x)=S^{-1}[x, U]$ for all $x \in A$. Writing $U$ in its Jordan form modulo a scalar, we can choose $U$ to be one of the following three types:

$$
\left(\begin{array}{lll}
0 & 1 & 0 \\
0 & 0 & 1 \\
0 & 0 & 0
\end{array}\right),\left(\begin{array}{lll}
0 & 1 & 0 \\
0 & 0 & 0 \\
0 & 0 & \gamma
\end{array}\right) \text { and }\left(\begin{array}{ccc}
\alpha & 0 & 0 \\
0 & 0 & 0 \\
0 & 0 & \gamma
\end{array}\right) \text { where } \alpha, \gamma \in \mathbf{C} \text { with } \alpha \neq 0
$$


Since the first two cases have the same argument, we choose $U=\left(\begin{array}{lll}0 & 1 & 0 \\ 0 & 0 & 1 \\ 0 & 0 & 0\end{array}\right)$ as an example. Let $S=\left(\begin{array}{lll}a_{11} & a_{12} & a_{13} \\ a_{21} & a_{22} & a_{23} \\ a_{31} & a_{32} & a_{33}\end{array}\right)$. Applying the fact that, for each $v \in E$, $S v, U v$ and $v$ are $\mathbf{C}$-dependent to $v=\left(\begin{array}{l}0 \\ 1 \\ 0\end{array}\right)$ and $\left(\begin{array}{l}1 \\ 1 \\ 0\end{array}\right)$ we see that $a_{32}=0=$ $a_{31}$. Thus $S^{-1}=\left(\begin{array}{lll}a & b & e \\ c & d & f \\ 0 & 0 & g\end{array}\right)$. Let $x=\left(\begin{array}{lll}1 & 0 & 0 \\ 0 & 0 & 0 \\ 0 & 0 & 0\end{array}\right)$ and $z_{n}=\left(\begin{array}{ccc}1 & 0 & 0 \\ n & 1 & 0 \\ 0 & 0 & 1\end{array}\right)$ for $n \geq 1$. Then $\left[z_{n}^{-1} x z_{n}, U\right]=\left(\begin{array}{ccc}n & 1 & 0 \\ 0 & -n & 0 \\ 0 & 0 & 0\end{array}\right)$, and so $\delta\left(z_{n}^{-1} x z_{n}\right)=S^{-1}\left[z_{n}^{-1} x z_{n}, U\right]=$ $\left(\begin{array}{ccc}n a & a-n b & 0 \\ n c & c-n d & 0 \\ 0 & 0 & 0\end{array}\right)$. Let $\mu_{n}, \nu_{n}, 0$ be the eigenvalues of $\delta\left(z_{n}^{-1} x z_{n}\right)$. Then $\mu_{n} \nu_{n}=$ $n a(c-n d)-n c(a-n b)=n^{2}(b c-a d) \neq 0$. This means $\lim _{n \rightarrow \infty} r\left(\delta\left(z_{n}^{-1} x z_{n}\right)\right)=\infty$, a contradiction.

We next consider the case $U=\left(\begin{array}{ccc}\alpha & 0 & 0 \\ 0 & 0 & 0 \\ 0 & 0 & \gamma\end{array}\right)$. Let $S=\left(\begin{array}{lll}a_{11} & a_{12} & a_{13} \\ a_{21} & a_{22} & a_{23} \\ a_{31} & a_{32} & a_{33}\end{array}\right)$. Applying the fact that, for each $v \in E, S v, U v$ and $v$ are $\mathbf{C}$-dependent to $v=\left(\begin{array}{l}1 \\ 1 \\ 0\end{array}\right)$ and $\left(\begin{array}{l}1 \\ 2 \\ 0\end{array}\right)$ we see that $a_{31}=0=a_{32}$. Thus $S^{-1}=\left(\begin{array}{lll}a & b & e \\ c & d & f \\ 0 & 0 & g\end{array}\right)$. Let $x=\left(\begin{array}{lll}0 & 1 & 0 \\ 0 & 0 & 0 \\ 0 & 0 & 0\end{array}\right)$ and $z_{n}=\left(\begin{array}{ccc}1 & 0 & 0 \\ n & 1 & 0 \\ 0 & 0 & 1\end{array}\right)$. Then $\left[z_{n}^{-1} x z_{n}, U\right]=\left(\begin{array}{ccc}0 & -\alpha & 0 \\ -n^{2} \alpha & 0 & 0 \\ 0 & 0 & 0\end{array}\right)$, and so

$$
\delta\left(z_{n}^{-1} x z_{n}\right)=S^{-1}\left[z_{n}^{-1} x z_{n}, U\right]=\left(\begin{array}{ccc}
-n^{2} b \alpha & -a \alpha & 0 \\
-n^{2} d \alpha & -c \alpha & 0 \\
0 & 0 & 0
\end{array}\right) .
$$

Let $\mu_{n}, \nu_{n}$ and 0 be the eigenvalues of $\delta\left(z_{n}^{-1} x z_{n}\right)$. Then $\mu_{n} \nu_{n}=n^{2} \alpha^{2}(b c-a d) \neq 0$, implying that $\lim _{n \rightarrow \infty} r\left(\delta\left(z_{n}^{-1} x z_{n}\right)\right)=\infty$, a contradiction.

(II) Suppose that $\operatorname{dim}_{\mathbf{C}} E=2$. In this case we may choose $U=\left(\begin{array}{ll}0 & 1 \\ 0 & 0\end{array}\right)$ or $\left(\begin{array}{ll}\alpha & 0 \\ 0 & 0\end{array}\right)$, where $0 \neq \alpha \in \mathbf{C}$. The two cases can be proved by the same arguments above.

Consider the next case: there exists $P \in \operatorname{End}_{\mathbf{C}}(E)$ an idempotent of rank 1 such that $\operatorname{dim} \operatorname{span}\{I-P,(I-P) S,(I-P) U\}=1$. We also assume that $I, U$ and $S$ are $\mathbf{C}$-independent. By the case above we may suppose that $\operatorname{dim}_{\mathbf{C}} E>$ 3. By assumption, there exist $\alpha, \beta \in \mathbf{C}$ such that $(I-P) S=\alpha(I-P)$ and $(I-P) U=\beta(I-P)$. Then $S=\alpha I+P(S-\alpha I)$ and $U=\beta I+P(U-\beta I)$. Set 
$Q_{1}=P(S-\alpha I)$ and $Q_{2}=P(U-\beta I)$. Then $\operatorname{rank}\left(Q_{i}\right) \leq 1$ for $i=1,2$. According to the $\mathbf{C}$-independence of $I, S$ and $U$ we have $Q_{1} \notin \mathbf{C}$. Thus there exists $v \in E$ such that $v$ and $Q_{1} v$ are $\mathbf{C}$-independent. Also, since $Q_{2} \notin \mathbf{C}$ and $\operatorname{rank}\left(Q_{2}\right)=1$, there exists $w \in E$ such that $Q_{2} w \neq 0$. Let $W$ be the $\mathbf{C}$-subspace of $E$ generated by $v, Q_{1} v, Q_{2} w$. Then $\operatorname{dim}_{\mathbf{C}} W \leq 3$. Note that $Q_{1} E=\mathbf{C} Q_{1} v$ and $Q_{2} E=\mathbf{C} Q_{2} w$. Thus $W$ is invariant under $S$ and $U$. We consider the subalgebra $A^{\prime}=\{x \in A \mid$ $\pi(x) W \subseteq W\}$. Then, for $\pi(x) \in \pi\left(A^{\prime}\right)$, we have $\pi \delta(x) W=S[\pi(x), U] W \subseteq W$. Moreover, $\mathrm{M}_{3}(\mathbf{C})$ is a homomorphic image of $\pi\left(A^{\prime}\right)$. To reduce the present case to the case that $\operatorname{dim}_{\mathbf{C}} E \leq 3$, we must only make a further remark: Let $a \in \operatorname{End}_{\mathbf{C}}(W)$ be invertible. Then, by the density theorem [13, Theorem 6.7], there exists an invertible $z \in A$ such that $\left.z\right|_{W}=a$ and hence $\left.z^{-1}\right|_{W}=a^{-1}$.

We now consider the last case: $I, U$ and $S$ are $\mathbf{C}$-dependent. If $S$ is a scalar, then $\pi \delta(x)=\left[\pi(x), S^{-1} U\right]=[\pi(x), T]$ for all $x \in A$. Suppose that $\pi \delta(A) \neq 0$. Then $T$ is not central in $\operatorname{End}_{\mathbf{C}}(E)$. Choose $\zeta \in E$ such that $\zeta$ and $T \zeta$ are $\mathbf{C}$-independent. By the density of $\pi(A)$ on $\operatorname{End}_{\mathbf{C}}(E)$, there exists $x \in A$ such that $\pi(x) \zeta=0$ and $\pi(x) T \zeta=\zeta$. On the other hand, by [13, Theorem 6.7] there exist invertible $z_{n} \in A$ for $n \geq 1$ such that $\pi\left(z_{n}\right) \zeta=\zeta$ and $\pi\left(z_{n}\right) T \zeta=n T \zeta$. We then compute

$$
\pi \delta\left(z_{n}^{-1} x z_{n}\right) \zeta=\left(\pi\left(z_{n}^{-1}\right) \pi(x) \pi\left(z_{n}\right) T-T \pi\left(z_{n}^{-1}\right) \pi(x) \pi\left(z_{n}\right)\right) \zeta=n \zeta,
$$

a contradiction. Suppose next that $S$ is not a scalar. Then $U=\alpha I+\beta S$ for some $\alpha, \beta \in \mathbf{C}$. Thus

$$
\pi \delta(x)=S^{-1}[\pi(x), U]=S^{-1}[\pi(x), \alpha I+\beta S]=\beta\left(S^{-1} \pi(x) S-\pi(x)\right)
$$

for all $x \in A$. We follow the argument of [3, Theorem 3.1]. Choose $v \in E$ such that $v$ and $S v$ are $\mathbf{C}$-independent. Suppose first that $S^{2} v, S v$ and $v$ are $\mathbf{C}$-independent. Choose $x \in A$ and invertible $z_{n} \in A$ for $n \geq 1$ [13, Theorem 6.7] such that

$$
\begin{gathered}
\pi(x) S v=S^{2} v, \pi(x) S^{2} v=v, \\
\pi\left(z_{n}\right) v=S v, \pi\left(z_{n}\right) S v=-\left(n S v+S^{2} v\right), \text { and } \pi\left(z_{n}\right) S^{2} v=v+n S^{2} v .
\end{gathered}
$$

We then compute

$$
\pi \delta\left(z_{n}^{-1} x z_{n}\right) v=\beta\left(S^{-1} \pi\left(z_{n}^{-1}\right) \pi(x) \pi\left(z_{n}\right) S-\pi\left(z_{n}^{-1}\right) \pi(x) \pi\left(z_{n}\right)\right) v=n \beta v,
$$

a contradiction. Suppose next that $S^{2} v \in \mathbf{C} S v+\mathbf{C} v$. Set $W=\mathbf{C} S v+\mathbf{C} v$. Then $\operatorname{dim}_{\mathbf{C}} W=2$ and $W$ is invariant under $S$. This case is then reduced to the first case. We now finish the proof.

Proof of Theorem 1.2. " $\Rightarrow$ ": Let $z \in A$ be invertible. By assumption, $\delta(z) \in$ $\operatorname{rad}(A)$ and so $z^{-1} \delta(z) \in \operatorname{rad}(A)$. Thus $r\left(z^{-1} \delta(z)\right)=0$.

" $\Leftarrow$ ": Suppose that $\sup \left\{r\left(z^{-1} \delta(z) \mid z \in A\right.\right.$ is invertible $\}<M$, where $M$ is a positive number. Let $a \in \operatorname{rad}(A)$; then $(1+a)^{-1}=1-a(1+a)^{-1}$. Then

$$
\begin{aligned}
M & >r\left((1+a)^{-1} \delta(1+a)\right)=r\left((1+a)^{-1} \delta(a)\right) \\
& =r\left(\left(1-a(1+a)^{-1}\right) \delta(a)\right)=r(\delta(a)),
\end{aligned}
$$

since $a(1+a)^{-1} \delta(a) \in \operatorname{rad}(A)$. For $x \in A$ and a positive integer $n$ we have

$$
M>r(\delta(n a x))=r(\phi(a) \delta(n x)+\delta(a) n x)=n r(\delta(a) x),
$$

implying that $r(\delta(a) x)=0$ and so $\delta(a) \in \operatorname{rad}(A)$. Thus $\delta(\operatorname{rad}(A)) \subseteq \operatorname{rad}(A)$, and so $\delta$ canonically induces a $\bar{\phi}$-derivation $\bar{\delta}$ on $\bar{A}=A / \operatorname{rad}(A)$. Note that $z \in A$ is invertible if and only if $\bar{z}=z+\operatorname{rad}(A)$ is invertible in $\bar{A}$. Also, $r\left(z^{-1} \delta(z)\right)=$ $r\left(\bar{z}^{-1} \bar{\delta}(\bar{z})\right)$. Thus we may reduce to the case that $A$ is semisimple. We just claim 
that $\delta=0$. By [ [6, Corollary 4.3], both $\phi$ and $\delta$ are continuous. Let $(\pi, E)$ be a continuous irreducible representation of $A$.

Case 1. $\delta$ is $E$-outer. Suppose first that $\phi$ is $E$-outer. Let $\xi \in E \backslash\{0\}$. Then, by Theorem 2.2, we can choose $y \in A$ such that $\pi \delta(y) \xi=\xi, \pi \phi(y) \xi=0$ and $\pi(y) \xi=0$. Suppose next that $\phi$ is $E$-inner. Write $\pi \phi(x)=S^{-1} \pi(x) S$ for all $x \in A$, where $S \in \operatorname{End}_{\mathbf{C}}(E)$ is invertible. If $S$ is a scalar, we choose an arbitrary $0 \neq \xi \in E$.

Otherwise, there exists $\xi \in E$ such that $S \xi$ and $\xi$ are $\mathbf{C}$-independent. In view of Theorem 2.1, there exists $y \in A$ such that $\pi \delta(y) \xi=\xi, \pi(y) S \xi=0$ and $\pi(y) \xi=0$. Thus $\pi \phi(y) \xi=0$ follows.

In either case, we define $z_{n}=e^{n y}$ for $n \geq 0$. Then each $z_{n}$ is invertible and $\pi \delta\left(z_{n}\right) \xi=n \xi, \pi\left(z_{n}\right) \xi=\xi$. So $\pi\left(z_{n}^{-1} \delta\left(z_{n}\right)\right) \xi=n \xi$, and hence $r\left(z_{n}^{-1} \delta\left(z_{n}\right)\right) \geq n$, a contradiction.

Case 2. $\delta$ is $E$-inner. We write $\pi \delta(x)=\pi \phi(x) T-T \pi(x)$ for all $x \in A$, where $T \in \operatorname{End}_{\mathbf{C}}(E)$.

Suppose that $\phi$ is $E$-outer. If $T$ is a scalar, by [3, Theorem 3.1] we choose $0 \neq \xi \in E$ and $z_{n} \in A$ invertible for $n \geq 1$ such that $\pi \phi\left(z_{n}\right) \xi=n \xi$ and $\pi\left(z_{n}\right) \xi=\xi$. Then

$$
\pi\left(z_{n}^{-1} \delta\left(z_{n}\right)\right) \xi=\pi\left(z_{n}^{-1}\right)\left(\pi \phi\left(z_{n}\right) T-T \pi\left(z_{n}\right)\right) \xi=\pi\left(z_{n}^{-1}\right) T((n-1) \xi)=(n-1) T \xi,
$$

a contradiction. Assume that $T$ is not a scalar. Choose $0 \neq \xi \in E$ such that $\xi$ and $T \xi$ are $\mathbf{C}$-independent. For $n \geq 0$ we choose an invertible $z_{n} \in A$ such that $\pi\left(z_{n}\right) \xi=\xi$ and $\pi \phi\left(z_{n}\right) T \xi=T \xi+n \xi$. Then $\pi\left(z_{n}^{-1} \delta\left(z_{n}\right)\right) \xi=n \xi$, implying that $r\left(z_{n}^{-1} \delta\left(z_{n}\right)\right) \geq n$. This is absurd.

Suppose next that $\phi$ is $E$-inner. Then $\pi \phi(x)=S^{-1} \pi(x) S$ for all $x \in A$, where $S \in \operatorname{End}_{\mathbf{C}}(E)$ is invertible. Write $\pi \delta(x)=S^{-1}[\pi(x), U]$, where $U=S T$. Suppose on the contrary that $\delta \neq 0$. Thus $U$ is not a scalar. Choose $\xi \in E$ such that $U \xi$ and $\xi$ are $\mathbf{C}$-independent. Then, for an integer $n \geq 0$, we can choose $\beta_{n}=1$ or 2 such that $\xi$ and $U \xi+n \beta_{n} S \xi$ are $\mathbf{C}$-independent. Thus, by [13 Theorem 6.7], there exist $z_{n} \in A$ invertible for $n \geq 0$ such that $\pi\left(z_{n}\right) \xi=\xi$ and $\pi\left(z_{n}\right) U \xi=U \xi+n \beta_{n} S \xi$. Then

$$
\pi\left(z_{n}^{-1} \delta\left(z_{n}\right)\right) \xi=\pi\left(z_{n}^{-1}\right) S^{-1}\left(\pi\left(z_{n}\right) U-U \pi\left(z_{n}\right)\right) \xi=n \beta_{n} \xi
$$

a contradiction. Thus $\delta=0$ follows. This proves the theorem.

\section{ACKNOWLEDGMENTS}

The research is supported by the NSC of R.O.C. (Taiwan).

\section{REFERENCES}

1. M. Brešar, Derivations decreasing the spectral radius, Arch. Math. 61 (1993), 160-162. MR 1230944 (94g:46046)

2. M. Brešar, Derivations of noncommutative Banach algebras, II, Arch. Math. 63 (1994), 56-59. MR 1277911 (95g:46086)

3. M. Brešar, On automorphisms of Banach algebras, Arch. Math. 78 (2002), 297-302. MR1895502 (2003a:46068)

4. M. Brešar and M. Mathieu, Derivations mapping into the radical, III, J. Functional Analysis 133 (1995), 21-29. MR.1351640 (96i:46054)

5. M. Brešar and P. Šemrl, On locally linearly dependent operators and derivations, Trans. Amer. Math. Soc. 351 (1999), 1257-1275. MF 1621729 (99e:47039)

6. M. Brešar and A.R. Villena, The noncommutative Singer-Wermer conjecture and $\phi-$ derivations, J. London Math. Soc. 66 (2002), 710-720. MR1934301 (2003h:46073) 
7. R. Curto and M. Mathieu, Spectrally bounded generalized inner derivations, Proc. Amer. Math. Soc. 123 (8) (1995), 2431-2434. MR1249873 (95j:46055)

8. B.E. Johnson, The uniqueness of the (complete) norm topology, Bull. Amer. Math. Soc. 73 (1967), 537-539. MR.0211260 (35:2142)

9. T.-K. Lee, Derivations on noncommutative Banach algebras, preprint.

10. C.-K. Liu, Extended Jacobson density theorem for rings with skew derivations, Ph.D. Thesis, Department of Mathematics, National Taiwan University, 2003.

11. M. Mathieu and G.J. Murphy, Derivations mapping into the radical, Arch. Math. 57 (1991), 469-474. MR1129522 (92j:46085)

12. V. Runde, Range inclusion results for derivations on noncommutative Banach algebras, Studia Math. 105 (1993), 159-172. MR1226626|(94h:46072)

13. A.M. Sinclair, Automatic continuity of linear operators, London Math. Soc. Lecture Note Ser. 21, Cambridge Univ. Press, 1976. MR0487371 (58:7011)

14. I.M. Singer and J. Wermer, Derivatons on commutative normed algebras, Math. Ann. 129 (1955), 260-264. MR0070061 (16:1125c)

15. M.P. Thomas, The image of a derivation is contained in the radical, Ann. of Math. 128 (1988), 435-460. MR0970607 (90d:46075)

16. Yu.V. Turovskii and V.S. Seul'man, Conditions for massiveness of the range of the derivation of a Banach algebra and associated differential operators, Math. Notes 42 (1987), 669-674. MR0915119 (89e:46051)

Department of Mathematics, National Taiwan University, Taipei 106, Taiwan

E-mail address: tklee@math.ntu.edu.tw

Department of Mathematics, National Taiwan University, Taipei 106, Taiwan

E-mail address: ckliu@math.ntu.edu.tw 